\title{
LETTERS
}

\section{Unmatched Canadian medical graduates}

The Canadian residency match is high stakes for Canadian medical students, as it is the only in-country route into clinical practice for Canadian medical graduates. There is an increasing number of unmatched medical graduates in Canada. This trend is of grave concern to the Canadian Federation of Medical Students, which represents more than 8000 medical students across Canada. The article by Drs. Wilson and Bordman, titled "What to do about the Canadian Residency Matching Service?", ${ }^{1}$ provides only a glimpse into the complexity of the residency matching system, and we fear that it may lead some readers astray.

We are concerned by the claim that Canadian medical graduates face direct competition from international medical graduates. In most provinces, Canadian and international medical graduates apply for separate positions during the first iteration of the match, and direct competition arises only in the second iteration. What is unclear from the available data is the number of Canadian medical graduates who applied for each secondround position. Some Canadian medical graduates in the second iteration might not apply to (or rank) positions, as a result of the availability of specialty positions, location of positions or other factors. One cannot simply look at the numbers of international versus Canadian medical graduate matches and draw an accurate conclusion.

The Canadian Federation of Medical Students supports increasing the ratio of 1.20 , which would facilitate greater student choice and flexibility in the system and result in fewer unmatched students. Even a number like this requires nuance; however, for example, one must consider position-specific language requirements. In 2017, 1.026 residency positions were available for every 1 graduate, but the ratio of anglophone positions to anglophoneonly applicants drops to 0.986 spots for every 1 student. The ratio of the numbers of medical students studying in each province to the number of residency seats also varies across the country, with not all provinces having adequate postgraduate seats. According to data from the Canadian Residency Matching Service (CaRMS), Saskatchewan had the lowest ratio at 0.879 in the 2017 match, and Nova Scotia was the only province to approach our recommended ratio of 1.20 , having the highest provincial ratio at 1.157 . The competitiveness of the system, resulting from tight and nondynamic ratios, is a substantial cause of distress to medical students, and largely responsible for our currently increasing numbers of unmatched students.

Furthermore, we are cautious of the comparison with European medical schools, many of which do not require previous university degrees for medical school admission. They exist within the context of primary university education, with students often entering directly from high school; these students experience different pressures and norms in university than students in Canadian medical schools do; and these schools have different residency requirements. The accumulation of student debt, as well as the different role of medical schools within Canadian society, must be considered in comparison with the European model.

Finally, it is important to consider the breadth of student motivations and circumstances in their match strategy; steps toward limiting the number of programs to which a student may apply may have meaningful consequences. For students intending to enter a "couples match" with a fellow colleague; for students hoping for a residency for which each program may have only a few seats; for students interested in more than one career path; or for students who need to apply before completing all core rotations, a limitation of applications could substantially harm the student's chance at a successful match.

The Canadian Federation of Medical Students is advocating for greater financial support from all organizations, including individual medical schools and postgraduate programs, to reduce the burden of the match. To address the financial burden associated with the final year of medical school better, efforts should be made to reduce cost per application, increase interview flexibility so as to minimize travel costs, and design a more objective selection process in which electives are valued less as auditions and more as opportunities for learning and broadening skills.

The Canadian Federation of Medical Students is working tirelessly with stakeholders and medical students across the country to address the "unmatched medical graduate crisis" in Canada.

\section{Sarah L. Silverberg}

Medical Student, University of Toronto

Faculty of Medicine, Toronto, Ont.;

Unmatched Canadian Medical Graduate

File Lead, Canadian Federation of

Medical Students

\section{Kaylynn M.H. Purdy}

Medical Student, Northern Ontario

School of Medicine, Thunder Bay, Ont.;

Vice-President Medical Education,

Canadian Federation of Medical Students

Cite as: CMAJ 2018 January 29;190:E118. doi: $10.1503 / \mathrm{cmaj} .68737$

\section{Reference}

1. Wilson CR, Bordman ZN. What to do about the Canadian Resident Matching Service. CMAJ 2017; 189:E1436-47.

Competing interests: The authors are both final-year medical students entering the R1 residency match in the 2017/2018 cycle. Sarah Silverberg is Unmatched Canadian Medical Graduate File Lead at the Canadian Federation of Medical Students, and Kaylynn Purdy is VicePresident Medical Education of the Canadian Federation of Medical Students. 